\title{
Research on Appeal of English Movie Title Translation based on Schema
}

\section{Theory}

\section{Dongmei Zhou}

\author{
School of foreign languages, Wuhan Textile University ,Hubei, China
}

Keywords: Movie Title Translation; Scheme Theory; Adaption Theory

\begin{abstract}
This paper aims to analyze translation strategies under the guidance of Adaption Theory. We picked out 100 movies with more than one title from the angle of different schemas to conduct the questionnaire on the basis of Schema theory. to find correlations between schema and appeal of movie titles. Based on data, we reach a conclusion that traditional culture and strange exotic culture have a negative correlation with appeal while familiar exotic culture and popular culture have a positive correlation with appeal. Furthermore, genre reflecting and explication have positive correlations with appeal as well.
\end{abstract}

\section{Introduction}

Nowadays, the translated English movie titles become the direct windows to know English movies. How to make the title appeal to Chinese audience? A lot of translators have conducted explorations about that. According to Nida (2001), movie title reflects the main ideas of a movie and plays a role in arising the audience's eager for watching this movie. It is of great importance to name a Chinese title before an English movie is released in China.

The concept of schema was firstly put forward by German Philosopher Kant which stands for the network of existing knowledge or experience stored in people's brains. Based on this, Swiss psychologist Piaget (1952) developed it into cognitive schema. This theory is widely applied in the fields of education and translation now.Schema own its unique characteristics including excitability, stability and adaptability. People manage to find connections of schema and new things to help them understand the new contents. At the same time, they will predict further information on the basis of those known contents and make a positive or negative judgment on the predictions when dealing with information. (Baker, 2004). Further studies also show that the process that audience get contact with movie titles and then generate interests to watch the movie is closely linked with schema. When a person gets contacts with a movie title, based on language schema, he understands the literal meaning of the title and then he arises some associations about the title which may have a direct influence on his interests. On contrast,if a movie title contains familiar content schema to audience,it may shorten the audience's thinking time and more easily draw their attention. Besides,schema usually have something to do with experience, knowledge, motives, interests, emotions and so on. Therefore, it is of great significance to find out what kind of content schema will stimulate audience's associations to induce their interests. And this kind of interests is what we call appeal.

Adaption Theory is a theory about the process of using language with the characteristics as follows: variability, negotiability adapt ability. It pointed out that language is process to adapt the context and actual intentions. (Verschueren, 2000). As for the movie title translation, only when movie titles comply with the audience's tastes can they appeal to the audience and inflame audience 
to have a watch. In this study, all the researches are on the basis of adaption, trying to find which kinds of movie titles adapt audience's tastes and making full use of these characteristics in movie title translation in the future to increase appeal of movie title.

\section{Research Design}

Research respondents. According to the Research Report on Chinese Film Industry in 2015 and Varity magazine,a well-known American movie magazine, the main force of today's Chinese film market are young audience whose average age is just 21.6 years old and their views have some certain references and guidance. What's more, the person in charge of Ai Qiyi, a Chinese leading video website, expressed his opinion in Chinese young audience. He regarded them as the focus of film industries for the reason that their reactions to movies have a large influence on public trends to watch movies.

The contents of the questionnaire. Considering that the appeal of movie titles must be universal, the questionnaire doesn't distinguish the specific territory, major, college, even the age at design time. But when it was distributed, we chose respondents from different places and with different backgrounds on purpose. The questionnaire is composed by three parts. The first part only contain the basic information regarding gender that we think it may have something to do with study. For the second part, the question is relevant to the favorite movie types for the reason that we want to know the audience'stastes. What's more, the last and most important part is a random test bank which consist of 100 movies, but every respondent just need to finish 20 movies presented randomly to pick out the most attractive movie title from three options.

The 100 movies are selected from 1263 movies collected from Douban Movie (Chinese biggest movie community online) which are divided into five universal types by us, including Comedy, Science Fiction, Suspense and Thriller, Romance, Action whose productions are top of all types. Generally speaking, production is determined by the market to some extent, so samples chosen from these movie types are relevantly more of values. Besides, due to the study on the correlations between schema and appeal,we try to screen movie titles in combination with schema and pick out several kinds of schema in those selected movie titles.

Table 1 Several Schema in Movie Titles

\begin{tabular}{|l|l|l|l|l|l|}
\hline & Exotic culture & Traditional culture & genre & Popular culture & explication \\
\hline Number of films & 66 & 24 & 557 & 19 & 110 \\
\hline Proportion of total films & $5.20 \%$ & $1.90 \%$ & $44.10 \%$ & $150 \%$ & $8.7 \%$ \\
\hline
\end{tabular}

As can be seen in the table, content schema and culture schema including traditional culture,foreign culture, popular culture as well as movie titles reflecting the genres and with explication are contained in movie titles, which can arouse audience associations in accordance with their experience and preference before.

Research procedure. Considering that the valid data thatevery question ought to be finished for at least 30 times, the simple size is at least 150 people. The questionnaire surveywas organized on May 12th, 2016 and May 13th, 2016. In order to finish the questionnaire survey quickly and efficiently, we sent the questionnaire only in WeChat, including the oriented Moments and WeChat 
groups. Therefore, the final target respondents of this study included college students from different places, young colleagues from the companies that I once interned in, some high school classmates or friends and young relatives who work in the factories. The questionnaire survey only took two hours to reach the double simple size and finally got 345 effective respondents including 168 males and 177 females.

\section{Findings and Discussions}

Owing to the definition of valid data---every question ought to be finished for at least 30 times, several data were of no use. Furthermore, some translation characteristics of movie titles which are put into the questionnaire at random, such as those involved with homophonic words are not so typical and also need to be ignored. Thus,the final valid data that we would have a discussion about are 63 movies. The specific proportional distributions and the overall chosen results are as follows:

Table 2 Proportional Distribution of Different Schema

\begin{tabular}{|l|l|l|l|l|l|}
\hline & Exotic culture & Traditional & Reflecting genre & Popular culture & explication \\
\hline numbers & 6 & 6 & 20 & 11 & 20 \\
\hline Elected movies & 3 & 2 & 15 & 9 & 18 \\
\hline Proportion of & $50 \%$ & $33.3 \%$ & $75 \%$ & $81.82 \%$ & $90 \%$ \\
\hline elected movies & & & & & \\
\hline
\end{tabular}

As a whole,schema have a positive correlation with appeal. In specific,movie titles involved with traditional culture are less attractive than those involved with other schema; movie titles involved with relevantly familiar schema have more appeal than those involved with strange schema; movie titles involved with the schema which can evoke more associations can gain more attractiveness than others.

\section{Summary}

Throughout previous studies, very few scholars focus on appeal of movie title translation from the perspective of schema. Nonetheless, in accordance with results achieved in this study, it turns out that schema indeed have great influence on appeal of movie title translation.Essentially speaking, appeal is just the positive reaction to the combination of what people think and what people see. It will be easier to generate attractiveness when movie titles establish connections with schema stored in audience's brains. And generally speaking, the more relates schema exist in movie titles, the more attractiveness they may have. According to the data, several conclusions can be reached as follows:

Culture schema play a vital role in appeal of movie title translation.Familiar culture schema have a positive correlation with appeal while strange culture schema have an opposite effect. To be specific, traditional culture schema such as movie titles with poetic style aren't to today's young 
audience' tastes while popular culture such as movie titles with current popular elements just appeal to them. In addition, some exotic culture also act the part of gaining audience's attractiveness.It is obvious that young audience today have few contacts to traditional culture,especially poems which are mainly learned from textbooks and relevantly not very straightforward to understand. The limited knowledge reserve of poems and pursuit of fast pace of life that audience want to know more movie contents as quickly as possible determine the less attractiveness in traditional culture schema.By the contrast, popular culture which is popular among people in current society has formed great schema in audience's brains before they see movie titles. Once movie titles involve these popular culture, it will be easier to evoke audience's schema and generate interests to explore further. So does the exotic culture. Familiar exotic culture gain more attractiveness in that it has already formed great schema in audience's brains to help audience know the contents while strange exotic culture is hard to establish connections with schema in audience's brains.

Content schema formed based on audience's experience and preferences have something to do with the appeal of movie titles as well. As mentioned in findings, it is very necessary to reflect genres in movie titles which will help audience take more associations about movie contents for the reason that long-term experiences and preferences of watching movies have formed schema in audience's brains that a certain genre of movies has its characteristics or plots which may help diminish their decision time and increase attractiveness to some degree.

Based on the second point above, it is also of great significance to evoke more schema in audience's brains by means of explication, which will help get rid of audience's fuzzy impressions and take more associations of movies to stimulate them explore further contents out of curiosity.

In addition, all the studies above are under the guidance of Adaption Theory because movie title translation is actually an intercultural activity which asks the translators to adapt the audience's preferences and demands. For movie titles, audience in different times have different tastes and translation strategies ought to be changeable in accordance with the characteristics of today's audience. It is worth doing research based on the data and surveys to know more about the target audience instead of studying with empty talk.

\section{References}

[1] Baker, Mona. Routledge. 2004,Encyclopedia of Translation Studies. Shanghai: Shanghai Foreign Language Education Press.

[2] Liu Biqing.1999, Contemporary Translation Theory[M].Beijing:Chinese Translation \&Publishing corporation.

[3]Nida. E.A. 2001, Language and culture: context in translation. Shanghai: Shanghai Foreign Language Education Press,

[4]Verschueren, J. 2000, Understanding Pragmatics. Beijing: Foreign Language Teaching and Research Press,.

[5]XuMin. 2015, Research on the Movie Title Translation Based on Schema Theory[J]. Communication. 